\title{
La réponse au stress osmotique des bactéries lactiques Lactococcus lactis et Lactobacillus plantarum (mini-revue)
}

\author{
Yves Romeo, Jean Bouvier, Claude GutiERREZ** \\ Laboratoire de Microbiologie et Génétique Moléculaire, Institut de Biologie Cellulaire et Génétique, \\ UMR 5100 du CNRS, 118 route de Narbonne, 31062 Toulouse Cedex, France
}

\begin{abstract}
Osmotic stress response of lactic acid bacteria Lactococcus lactis and Lactobacillus plantarum. In order to survive in a wide variety of environments, bacteria have evolved systems that protect themselves against environmental stress. Lactic acid bacteria grow in media where osmolarity is high and varies frequently and they must adjust their intracellular osmolarity in order to maintain the turgor pressure necessary for cell elongation. An osmotic upshock stops their growth and activates specific mechanisms which prevent cells death. The regulatory mechanisms of the response of Lactococcus lactis and Lactobacillus plantarum to an osmotic stress have drawn increasing attention in recent years, because of their use in industrial fermentations and their fundamental interest as Gram positive bacteria. The main response to osmotic stress is the accumulation in the cytoplasm of osmoprotectant organic compounds, the so-called "compatible solutes", which can accumulate to very high levels without deleterious effects on the cellular metabolism. In this review, we present the state of the art on the physiological and molecular responses of L. lactis and L. plantarum to an osmotic stress.
\end{abstract}

osmotic regulation / lactic acid bacteria / stress / ABC transporter

Résumé - Les bactéries sont capables de vivre dans des milieux extrêmement variés car elles possèdent des systèmes de protection efficaces contre les différents stress qu'elles peuvent rencontrer. Les bactéries lactiques vivent dans des habitats où l'osmolarité est souvent élevée et varie énormément, alors que la pression osmotique intracellulaire doit rester relativement constante. Lorsqu'une bactérie subit un stress osmotique, dû à une forte augmentation de la concentration en sel dans l'environnement, sa croissance est arrêtée, et des mécanismes de détresse se mettent en place pour éviter la mort cellulaire. Les études sur la réponse au stress osmotique des bactéries lactiques Lactococcus lactis et Lactobacillus plantarum et les mécanismes moléculaires sous-jacents se sont développées depuis ces dernières années, du fait de l'intérêt économique (utilisation dans l'industrie agroalimentaire) et fondamental (bactéries à Gram positif) que représentent ces organismes. La principale réponse à un choc osmotique consiste en l'accumulation dans le cytoplasme de molécules protectrices,

* Correspondance et tirés-à-part

Tél. : (33) 561335872 ; fax : (33) 561335886 ; e-mail : clg @ ibcg.biotoul.fr 
appelées solutés compatibles, qui permettent le rétablissement de l'équilibre osmotique à l'intérieur de la bactérie. Dans cette revue, nous présenterons les connaissances actuelles sur la réponse au stress osmotique de L. lactis et L. plantarum au niveau physiologique et moléculaire.

régulation osmotique / bactérie lactique / stress / $\mathrm{ABC}$ transporteur

\section{INTRODUCTION}

Dans leur habitat naturel, les micro-organismes sont fréquemment exposés à des variations de pression osmotique du milieu environnant. La membrane cytoplasmique des bactéries est perméable à l'eau mais constitue une barrière efficace contre le passage de la plupart des solutés du milieu et des métabolites présents dans le cytoplasme. Une augmentation brusque de l'osmolarité du milieu externe entraîne un rapide flux d'eau vers l'extérieur de la cellule, qui a pour conséquence une diminution de la pression de turgescence, le moteur de l'élongation des cellules, une variation de la concentration cytoplasmique en solutés et un changement du volume cellulaire (plasmolyse dans les cas extrêmes). À l'inverse, un choc hypotonique provoque une entrée d'eau dans la bactérie et une augmentation du volume cytoplasmique et de la pression de turgescence. De telles variations osmotiques étant délétères pour les bactéries, celles-ci possèdent des systèmes de transport et/ou de synthèse d'osmoprotectants ou solutés compatibles qui assurent le maintien de l'homéostasie par leur accumulation dans le cytoplasme ou leur rejet selon le type de stress osmotique $[4,5,17]$. Les solutés compatibles sont des composés organiques qui peuvent être accumulés à forte concentration dans le cytoplasme sans interférer avec les processus cellulaires, la molécule majeure étant la glycine bétaïne (N,N,N-trimethylglycine), qui appartient à la famille des composés ammonium quaternaires [8].

Dans leur environnement naturel, les bactéries lactiques peuvent être soumises à de forts stress osmotiques, causés par exemple par la dessiccation. Il en est de même lors de leur utilisation dans les processus de fermentation industriels, par exemple lors de la salaison des caillés au cours de la fabrication des fromages. L'étude des mécanismes de protection contre le stress osmotique chez ces bactéries est donc d'un intérêt fondamental pour la compréhension des mécanismes de défense contre le stress osmotique chez les bactéries à Gram positif, mais aussi nécessaire pour une utilisation optimale de celles-ci dans les procédés industriels. Les données actuelles sur la réponse des bactéries lactiques au stress osmotique concernent essentiellement L. lactis et $L$. plantarum, auxquelles nous nous intéresserons ici.

\section{RÉPONSE PHYSIOLOGIQUE}

Une augmentation de la concentration en sel dans le milieu externe entraîne un arrêt de croissance des bactéries. Ainsi, la croissance de L. lactis dans un milieu à 2,5\% NaCl (concentration proche de celle rencontrée dans certains fromages) est réduite de $25 \%$ à $50 \%$ par rapport à une croissance à pression osmotique normale [18]. Chez les bactéries à Gram négatif Escherichia coli et Salmonella typhymurium, la réponse initiale à une augmentation de la pression osmotique du milieu environnant est une accumulation d'ions $\mathrm{K}^{+}$dans le cytoplasme via les transporteurs à faible et haute affinité pour le potassium, respectivement nommés Trk et Kdp [19, 31, 32]. Afin de maintenir l'électroneutralité du milieu intracellulaire, cette accumulation s'accompagne d'une synthèse de novo de glutamate [24]. Le 
glutamate de potassium est ensuite progressivement remplacé par des composés plus compatibles avec les processus physiologiques de la cellule [6], le principal étant la glycine bétaïne. Certaines bactéries sont capables de synthétiser cette molécule, soit par méthylation de la glycine, soit par oxydation de la choline [20,34]. Cependant, la plupart possèdent et utilisent des systèmes de transport qui permettent une importation rapide et en masse de ce composé depuis le milieu environnant, le mieux caractérisé étant le système de transport proU à haute affinité pour la glycine bétaïne d' E. coli [3, $7,13,23]$.

Chez les bactéries lactiques, la réponse à un choc hyperosmotique diffère légèrement de celle des bactéries à Gram négatif. En effet, le cytoplasme des bactéries lactiques présente en condition normale de culture une concentration en potassium très élevée (environ $1 \mathrm{~mol} \cdot \mathrm{L}^{-1}$ ), ainsi qu'un important pool d'acides aminés, le plus abondant étant le glutamate [9, 12, 25, 28]. Des études récentes chez L. plantarum ont montré que, contrairement aux Entérobactéries, la majeure partie du potassium se trouve à l'état lié dans la cellule et qu'il n'aurait donc peut-être pas de rôle majeur dans le maintien de l'équilibre osmotique de ces cellules $[9,12]$. Cette hypothèse est renforcée par le fait que l'augmentation de la concentration cytoplasmique en potassium en réponse à un choc hypertonique n'est observée que lorsque l'augmentation de la salinité du milieu environnant est provoquée par du $\mathrm{KCl}$ et non du $\mathrm{NaCl}$, suggérant que le transport des ions $\mathrm{K}^{+} \mathrm{n}^{\prime}$ intervient pas en réponse à l'augmentation de la pression osmotique. La réponse initiale des bactéries lactiques à une augmentation de pression osmotique dans le milieu environnant correspond à une accumulation de proline et de glycine bétaïne dans leur cytoplasme $[10,26]$. Des mesures de croissance ont montré que la glycine bétaïne et, dans une moindre mesure la proline, sont capables de protéger $L$. lactis contre un stress osmotique [26, 27]. Par contre, l'ectoïne, la carnitine, la taurine et la choline ne confèrent pas de protection contre une forte osmolarité [27]. À la différence des Entérobactéries et de Bacillus subtilis, les bactéries lactiques ne présenteraient pas de voie de biosynthèse de solutés compatibles. Ainsi, L. plantarum n'est pas capable de synthétiser la glycine bétaïne, et l'accumulation rapide de ce soluté dans le milieu intracellulaire observée en réponse à un choc hyperosmotique est due exclusivement au transport de ce composé depuis le milieu extérieur vers le cytoplasme. Des travaux ont mis en évidence l'existence chez cette espèce bactérienne d'un système de transport à haute affinité pour la glycine bétaïne et la carnitine et à basse affinité pour la proline, nommé QacT (Quaternary ammonium compound Transporter) [11]. Une augmentation de l'activité de transport de la glycine bétaïne via QacT a été observée spécifiquement lors d'une élévation de la concentration en sel du milieu extérieur et constituerait, pour L. plantarum, la réponse initiale à un choc hypertonique. Des études chez L. lactis ont aussi démontré l'existence d'un opéron codant un système de transport à haute affinité pour la glycine bétaïne appelé BusA (betaine uptake system A) ou OpuA (osmoprotectant uptake A), dont l'expression augmente spécifiquement lors d'un choc hypertonique [2, 27, 36]. OpuA de L. lactis appartient à la famille des transporteurs de type ABC (ATP Binding Cassette). De nombreuses études ont été effectuées sur cette famille de transporteurs que l'on retrouve parmi une large variété d'organismes procaryotes et eucaryotes [14]. Chez E. coli, l'ABC transporteur ProU est localisé au niveau de l'enveloppe et est composé de 3 protéines : une protéine cytoplasmique d'hydrolyse de l'ATP (ATPase ou ATPbinding cassette, ProV), caractéristique des $\mathrm{ABC}$ transporteurs, qui fournit l'énergie au système, une protéine membranaire (ou perméase, ProW) servant au passage de la glycine bétaïne vers le milieu interne et un polypeptide périplasmique hydrophile de liaison au substrat (ProX). La perméase ProW est constituée de 6 domaines transmembranaires 
aux extrémités carboxy- et amino-terminale cytoplasmiques et semble fonctionner comme un homodimère avec l'ATPase ProV [1, 23]. Chez L. lactis, l'étude de la séquence de l'opéron opuA indique que ce système appartient à la superfamille des ABC transporteurs. Cependant, elle révèle une organisation nouvelle du système. En effet, opuA n'est constitué que de 2 gènes. Le premier, ориAA, code une ATPase homologue de ProV. Le second, opuABC, code une protéine homologue à ProW dans sa partie amino-terminale et à ProX dans sa partie carboxy-terminale. Ainsi, la perméase OpuAB et la protéine de liaison au substrat OpuAC auraient fusionné pour donner une protéine hybride possédant à la fois les fonctions de liaison et de transport de la glycine bétaïne [2, 27]. Ces données basées sur une analyse de séquence ont été vérifiées par des analyses biochimiques de la structure de ce transporteur [27].

Ainsi, les bactéries lactiques ont développé des moyens de protection contre les stress osmotiques qui, bien qu'adaptés aux milieux dans lesquels elles évoluent, sont très semblables à ceux utilisés par les bactéries à Gram négatif, du fait de l'utilisation du même osmoprotectant majeur, la glycine bétaïne.

\section{RÉGULATION GÉNÉTIQUE}

En réponse aux chocs osmotiques, les bactéries ont développé des systèmes de défense efficaces qui reposent essentiellement sur l'accumulation de solutés compatibles dans leur cytoplasme. L'activation de ces systèmes nécessite que les cellules perçoivent les variations d'osmolarité de l'environnement et que l'information soit alors transmise et convertie en un signal qui déclenche une réponse au niveau moléculaire. Le premier point, la perception du signal osmotique, reste très mal compris. La deuxième étape de la réponse à un choc hypertonique est le traitement du signal osmotique qui aboutit au déclenchement d'une réponse moléculaire. La régulation des gènes codant les transporteurs des solutés compatibles a été très étudiée chez E. coli, en particulier celle de l'opéron $p r o U$ [21]. Aucun régulateur spécifique de l'expression de proU n'a été identifié à ce jour et il a été proposé que celle-ci serait assurée par un phénomène plus global. En effet, des résultats obtenus à partir de systèmes reconstitués in vitro ont suggéré que le glutamate de potassium stimulerait l'expression de proU lors d'une augmentation de la pression osmotique environnante, ceci par une action directe des ions $\mathrm{K}^{+}[16$, $29,30,33]$. Cette hypothèse très attractive, du fait que chez cette bactérie l'accumulation de glutamate de potassium correspond à la réponse initiale à un choc osmotique, ne suffit cependant pas à expliquer la très forte induction de l'expression de cet opéron, et d'autres travaux ont démontré que les variations de surenroulement de l'ADN en fonction de la concentration saline jouent un rôle dans l'osmorégulation de proU [15]. Enfin, il a été mis en évidence que le régulateur global H-NS est un répresseur de l'expression de $p r o U$ à faible pression osmotique, et que cet effet serait modulé par le glutamate de potassium et le surenroulement de l'ADN $[22,35]$.

L'étude de la réponse moléculaire des bactéries lactiques au stress osmotique a été entreprise chez L. plantarum et L. lactis. Chez L. plantarum l'expression du système de transport QacT est semi-constitutive et indépendante des conditions d'osmolarité [11] ; la régulation osmotique se situerait au niveau de l'activité du transporteur. Des mesures de cinétique de transport de la glycine bétaïne et de la proline effectuées à forte et faible osmolarité indiquent que l'accroissement de la vitesse de transport de ces deux solutés serait due à une augmentation de l'activité de QacT et non pas de son expression. L'activité de ce système serait en partie contrôlée par la proline et la glycine bétaïne. En effet, des cellules précultivées dans un milieu contenant de la proline puis soumises à un choc osmotique 
présentent un taux de transport de glycine bétaïne moins élevé que des cellules n'ayant pas subi le prétraitement ; ce résultat ainsi que d'autres tendent à montrer que la proline et la glycine bétaïne réprimeraient l'activité du système QacT. Cette inhibition semble être levée par une augmentation de l'osmolarité, cependant les travaux effectués ne permettent pas de l'affirmer.

L'analyse de la production de protéines en réponse à un choc hypertonique chez L. lactis a montré une diminution de la synthèse totale de protéines d'environ $50 \%$ lorsque les bactéries sont cultivées dans un milieu à 2,5\% $\mathrm{NaCl}$ [18]. Cependant, la synthèse d'au moins douze protéines est augmentée dans de telles conditions. Parmi celles-ci se trouvent des protéines impliquées dans la réponse à d'autres stress, comme les protéines DnaK, GroEL et GroES impliquées dans la réponse au choc thermique. Les autres protéines n'ont pour la plupart pas été caractérisées, cependant, certaines d'entre elles pourraient correspondre aux protéines OpuAA et OpuABC. En effet, la transcription de l'opéron opuA est induite lors d'une augmentation de la salinité du milieu extracellulaire. Ce résultat suggère l'existence d'une ou plusieurs protéines capables de contrôler spécifiquement l'expression des composants de ce transporteur en fonction de l'osmolarité du milieu. Une recherche de régulateurs de l'opéron opuA dans notre laboratoire a permis de mettre en évidence l'existence d'une protéine, nommée OpuR, capable de réprimer spécifiquement l'expression du promoteur d'opuA (résultats non publiés). Un mutant du gène opuR présente une augmentation de l'expression d'opuA à faible osmolarité, qui est presque complètement constitutive vis-à-vis de la concentration en $\mathrm{NaCl}$. Nos résultats suggèrent donc que le gène $o p u R$ code une protéine impliquée dans la régulation osmotique d'opuA. L'étude de la séquence protéique de OpuR indique qu'elle possède un domaine homologue à des transporteurs putatifs d'ions $\mathrm{K}^{+}$. Cette protéine pourrait ainsi être sensible à la concentration ionique du cytoplasme et réprimer ou non $о р и A$ en fonction de ce paramètre. Une analyse biochimique d'OpuR, en cours dans notre laboratoire, permettra de tester cette hypothèse.

La régulation osmotique d'opuA semble principalement porter sur l'expression de l'opéron, même si des données suggèrent aussi l'existence d'un contrôle au niveau de l'activité du transporteur [36]. La poursuite de l'étude de la régulation osmotique de opuA par OpuR permettra une meilleure compréhension des mécanismes moléculaires de la réponse à un choc osmotique chez L. lactis.

Alors qu'elles utilisent les mêmes molécules osmoprotectrices et les mêmes types de transporteurs en réponse à un choc osmotique, L. plantarum et L. lactis ont développé des mécanismes de régulation des systèmes de transports des solutés compatibles différents reposant pour l'une sur un contrôle de l'activité du transporteur, pour l'autre sur un contrôle de son expression.

\section{CONCLUSION}

L'adaptation des micro-organismes aux variations de pression osmotique du milieu environnant repose sur une accumulation de solutés compatibles dans le cytoplasme permettant leur croissance dans un milieu à forte concentration saline. Les mécanismes moléculaires impliqués dans cette réponse, en particulier l'activation des différents systèmes de transports de solutés compatibles, ont été intensivement étudiés chez les entérobactéries, E. coli et $S$. typhimurium principalement. Chez les bactéries lactiques, les études ont porté essentiellement sur l'adaptation physiologique des cellules en réponse à ces stress et peu d'informations existent sur les processus cellulaires engagés. Les protéines senseurs ainsi que les mécanismes de perception des signaux environnementaux et de transmission de l'information sont inconnus chez L. lactis et L. plantarum. Par ailleurs, aucun travail n'indique l'existence 
de facteurs sigma alternatifs qui pourraient intervenir dans la réponse au stress, comme c'est le cas chez B. subtilis. L'étude des processus moléculaires d'adaptation à une augmentation de l'osmolarité chez des organismes comme L. lactis, utilisés dans des processus industriels, tels que la fabrication des fromages à pâte molle, est importante d'un point de vue fondamental et économique. Les données actuelles et surtout à venir sur la régulation du système majeur de transport de la glycine bétaïne, OpuA de L. lactis pourront apporter des éléments de réponse sur le fonctionnement moléculaire de l'adaptation au choc osmotique chez les bactéries lactiques, et plus généralement chez les bactéries à Gram-positif. Par ailleurs, la compréhension de ces mécanismes présente un intérêt économique, qui consisterait en la construction d'outils biotechnologiques, tels que des vecteurs d'expression contrôlables par l'osmolarité, qui seraient utilisés dans divers procédés de l'industrie agroalimentaire.

\section{RÉFÉRENCES}

[1] Barron A., Jung J.U., Villarejo M., Purification and characterization of a glycine betaine binding protein from Escherichia coli, J. Biol. Chem. 262 (1987) 11841-11846.

[2] Bouvier J., Bordes P., Romeo Y., Fourçans A. Bouvier I., Gutierrez C., Characterization of OpuA, a glycine-betaine uptake system of $\mathrm{Lac}$ tococcus lactis, J. Mol. Microbiol. Biotechnol. 2 (2000) 199-205.

[3] Cairney J., Booth I.R., Higgins C.F., Osmoregulation of gene expression in Salmonella typhimurium: proU encodes an osmotically induced betaine transport system, J. Bacteriol. 164 (1985) 1224-1232.

[4] Csonka L.N., Physiological and genetic responses of bacteria to osmotic stress, Microbiol. Rev. 53 (1989) 121-147.

[5] Csonka L.N., Hanson A.D., Prokaryotic osmoregulation: genetics and physiology, Annu. Rev. Microbiol. 45 (1991) 569-606.

[6] Dinnbier U., Limpinsel E., Schmid R., Bakker E.P., Transient accumulation of potassium glutamate and its replacement by trehalose during adaptation of growing cells of Escherichia coli K12 to elevated sodium chloride concentrations, Arch. Microbiol. 1 (1988) 348-357.
[7] Dunlap V.J., Csonka L.N., Osmotic regulation of L-proline transport in Salmonella typhimurium, J. Bacteriol. 163 (1985) 296-304.

[8] Galinski E-A., Trüper H.G., Microbiol behaviour in salt-stressed ecosystems, FEMS Microbiol. Rev. 15 (1994) 95-108.

[9] Glaasker E., Konings W.N., Poolman B., Osmotic regulation of intracellular solute pools in Lactobacillus plantarum, J. Bacteriol. 178 (1996) 575-582.

[10] Glaasker E., Konings W.N., Poolman B., Glycine betaine fluxes in Lactobacillus plantarum during osmostasis and hyper- and hypoosmotic shock, J. Biol. Chem. 271 (1996) 10060-10065.

[11] Glaasker E., Heuberger E.H., Konings W.N., Poolman B., Mechanism of osmotic activation of the quaternary ammonium compound transporter (QacT) of Lactobacillus plantarum, J. Bacteriol. 180 (1998) 5540-5546.

[12] Glaasker E., Tjan F.S., Ter Steeg P.F., Konings W.N., Poolman B., Physiological response of Lactobacillus plantarum to salt and nonelectrolyte stress, J. Bacteriol. 180 (1998) 4718-4723.

[13] Gowrishankar J., Nucleotide sequence of the osmoregulatory proU operon of Escherichia coli, J. Bacteriol. 171 (1985) 1923-1931.

[14] Higgins C.F., ABC transporters: from microorganisms to man, Annu. Rev. Cell Biol. 8 (1992) 67-113.

[15] Higgins C.F., Dorman C.J., Stirling D.A., Waddell L., Booth I.R., May G., Bremer E., A physiological role for DNA supercoiling in the osmotic regulation of gene expression in $S$. typhimurium and E. coli, Cell 52 (1988) 569-584.

[16] Jovanovich S.B., Record M.T. Jr., Burgess R.B., In an Escherichia coli coupled transcriptiontranslation system, expression of the osmoregulated gene proU is stimulated at elevated potassium concentrations and by an extract from cells grown at high osmolarity, J. Biol. Chem. 264 (1989) 7821-7825.

[17] Kempf B., Bremer E., Uptake and synthesis of compatible solutes as microbial stress responses to high-osmolality environments, Arch. Microbiol. 170 (1998) 319-330.

[18] Kilstrup M., Jacobsen S., Hammer K., Vogensen F.K., Induction of heat shock proteins DnaK, GroEL and GroES by salt stress in Lactococcus lactis, Appl. Environ. Microbiol. 63 (1997) 1826-1837.

[19] Laimins L., Rhoads D., Epstein W., Osmotic control of $k d p$ operon expression in Escherichia coli, Proc. Natl. Acad. Sci. USA 78 (1981) 464-468.

[20] Landfald B., Strom A.R., Choline-glycine betaine pathway confers a high level of osmotic tolerance in Escherichia coli, J. Bacteriol. 165 (1986) 849-855. 
[21] Lucht J.M., Bremer E., Adaptation of Escherichia coli to high osmolarity environments: osmoregulation of the high-affinity glycine betaine transport system proU, FEMS Microbiol. Rev. 14 (1994) 3-20.

[22] Lucht J.M., Dersch P., Kempf B., Bremer E., Interactions of the nucleoid-associated DNAbinding protein $\mathrm{H}-\mathrm{NS}$ with the regulatory region of the osmotically controlled proU operon of Escherichia coli, J. Biol. Chem. 269 (1994) 6578-6586.

[23] May G., Faatz E., Lucht I., Haardt M., Bolliger M., Bremer E., Characterization of the osmoregulated Escherichia coli proU operon and identification of ProV as a membrane-associated protein, Mol. Microbiol. 3 (1989) 1521-1531.

[24] McLaggan D., Naprstek J., Buurman E.T., Epstein W., Interdependence of $\mathrm{K}+$ and glutamate accumulation during osmotic adaptation of Escherichia coli, J. Biol. Chem. 269 (1994) 1911-1917.

[25] Measures J.C., Role of amino acids in osmoregulation in non-halophilic bacteria, Nature 257 (1975) 398-400.

[26] Molenaar D., Hagting A., Alkema H., Driessen A.J., Konings W.N., Characteristics and osmoregulatory roles of uptake systems for proline and glycine betaine in Lactococcus lactis, J. Bacteriol. 175 (1993) 5438-5444

[27] Obis D., Guillot A., Gripon J.-C., Renault P., Bolotin A., Mistou M.-Y., Genetic and biochemical characterization of a high-affinity betaine uptake system (BusA) in Lactococcus lactis reveals a new functional organization within bacterial $\mathrm{ABC}$ transporters, J. Bacteriol. 181 (1999) 6238-6246.

[28] Poolman B., Hellingwerf K.J., Konings W.N., Regulation of the glutamate-glutamine transport system by intracellular $\mathrm{pH}$ in Streptococcus lactis, J. Bacteriol. 169 (1987) 2272-2276.
[29] Prince W.S., Villarejo M.R., Osmotic control of proU transcription is mediated through direct action of potassium glutamate on the transcription complex, J. Biol. Chem. 265 (1990) 17673-17679.

[30] Ramirez R.M., Prince W.S., Bremer E., Villarejo M., In vitro reconstitution of osmoregulated expression of proU of Escherichia coli, Proc. Natl. Acad. Sci. USA 86 (1989) 1153-1157.

[31] Schlosser A., Kluttig S., Hamann A., Bakker E.P., Subcloning, nucleotide sequence and expression of trkG, a gene that encodes an integral membrane protein involved in potassium uptake via the Trk system of Escherichia coli, J. Bacteriol. 173 (1991) 3170-3176.

[32] Schlosser A., Meldorf M., Stumpe S., Bakker E.P., Epstein W., TrkH and its homolog, TrkG, determine the specificity and kinetics of cation transport by the Trk system of Escherichia coli, J. Bacteriol. 177 (1995) 1908-1910.

[33] Sutherland L., Cairney J., Elmore M.J., Booth I.R., Higgins C.F., Osmotic regulation of transcription: induction of the $p r o U$ betaine transport gene is dependent on accumulation of intracellular potassium, J. Bacteriol. 168 (1986) 805-814.

[34] Trüpper H.G., Galinski E.A., Biosynthesis and fate of compatible solutes in extremely halophilic phototrophic eubacteria, FEMS Microbiol. Rev. 75 (1990) 247-254.

[35] Ueguchi C., Mizuno T., The Escherichia coli nucleoid protein $\mathrm{H}-\mathrm{NS}$ functions directly as a transcriptional repressor, EMBO J. 12 (1993) 1039-1046.

[36] van der Heide T., Poolman B., Glycine betaine transport in Lactococcus lactis is osmotically regulated at the level of expression and translocation activity, J. Bacteriol. 182 (2000) 203-206. 